\title{
Experimentación Sobre los Efectos de la Meteorización en la Supervivencia de Elementos Óseos de Percichthys trucha: Implicaciones Ictioarqueológicas para el Sitio DV1, Lago Musters (Prov. Chubut, Argentina)
}

Experimentation on the Weathering Effects of Percichthys trucha Bones' Survival: Archaeoichthyological Implications for DV1 Site, Musters Lake (Chubut, Argentina)

Ariadna Svoboda ${ }^{\mathrm{i}}$ y Eduardo J. Moreno ${ }^{\mathrm{ii}}$

\section{RESUMEN}

La representación de partes anatómicas de peces es un criterio ampliamente utilizado para determinar el origen antrópico o natural de los conjuntos ictiofaunísticos. Uno de los factores tafonómicos que suelen afectar la supervivencia de los elementos es la meteorización. Para evaluar los efectos de este proceso, se realizó una experimentación que consistió en la exposición a condiciones subaéreas de cuatro percas (Percichthys trucha) y varios huesos de oveja (Ovis aries) durante seis años. Los resultados obtenidos señalan que: I) existe supervivencia diferencial de partes esqueletales, la cual no es mediada por la densidad ósea; 2) en términos comparativos, los elementos óseos de peces se destruyen más rápido que los de mamíferos. Finalmente, al comparar estos resultados con el conjunto ictioarqueológico de Delta del Arroyo Vulcana I (lago Musters, Chubut) se infiere un bajo impacto de la meteorización, probablemente por un rápido enterramiento.

Palabras clave: Meteorización Ósea, Peces, Supervivencia Diferencial

\section{ABSTRACT}

Fish body part frequency is a widely used criteria to determine the cultural or natural origin of fish assemblages. Weathering is an important taphonomic process affecting elements survival. To assess the effects of this process an experiment was conducted. It's consists on the exposure of four perch (Percichthys trucha) and sheep bones (Ovis aries) for six years. The results show that: $I$ ) there is differential survival on body parts, not mediated by bone density; 2) in comparative terms, fish bone elements are destroyed faster than mammals. Finally, by comparing these results to those of Delta del ArroyoVulcana I fish assemblages (Musters Lake, Chubut) it's inferred a low impact of weathering, most likely by a rapid burial.

Key Words: Bone Weathering, Fish Bones, Differential Survival

\footnotetext{
i Centro Nacional Patagónico, CONICET. Boulevard Brown 2915, Puerto Madryn (9120), Chubut, Argentina. Correo-e: svoboda@cenpat. edu.ar

ii FHyCS, Universidad Nacional de la Patagonia San Juan Bosco. Km 4, Comodoro Rivadavia, Chubut, Argentina. Correo-e: moreno@cenpat. edu.ar
}

Recibido: 25-11-2012 - Revisado: 09-07-2013 - Aceptado: 22-10-2013 


\section{INTRODUCCIÓN}

Uno los criterios tafonómicos empleados para determinar el origen antrópico o natural de los conjuntos ictiofaunísticos es la representación de partes anatómicas (Acosta et al. 2007, Butler 1993, Gifford-Gonzalez et al. 1999, Stewart y GiffordGonzalez 1994, Zohar et al. 200I, entre otros). Se han evaluado varios agentes y procesos tafonómicos que afectan la representación de partes esqueletales; por ejemplo, actividades culturales (Butler 1993, GiffordGonzalez et al. 1999, Stewart y Gifford-Gonzalez 1994); termoalteración (Ritcher 1986); procesos digestivos (Nicholson 1992-1993); procesos diagenéticos de pisoteo y abrasión mecánica (Nicholson 1992a, 1992b), entre otros. En términos generales, son exiguos los estudios sobre los efectos de meteorización subaérea en peces. Algunas excepciones son las observaciones sobre restos de siluriformes en África oriental que luego de 10 años de exposición evidenciaban un avanzado grado de meteorización (Gifford-Gonzalez et al. 1999); y un experimento que consistió en la exposición de un bacalao durante 109 semanas en Europa del Norte (Nicholson 1991, en Nicholson 1992b).

La meteorización puede ser un factor crítico para la supervivencia de restos ícticos depositados en ambientes abiertos de Patagonia. Las observaciones en mamíferos y aves demuestran que la secuencia de destrucción ósea por causa de este proceso es relativamente rápida (Borrero 2007; Cruz 2008). En consecuencia, si consideramos que los huesos de pequeños animales -como los peces- se meteorizan más rápido que los de mayor porte es esperable un sesgo por efecto del tamaño (Behrensmeyer 1978).

Por lo expuesto, y teniendo en cuenta los hallazgos de perca (Percichthys trucha) en los sitios arqueológicos del lago Musters (Chubut, Argentina) (Moreno y Pérez Ruiz 20I0), surge la necesidad de indagar sobre los efectos de la meteorización en los conjuntos ícticos. El objetivo de este trabajo es generar un corpus de datos inicial que permita detectar e interpretar la acción de dicho proceso en la supervivencia diferencial de elementos anatómicos; otro de los objetivos es aproximarse a un mejor entendimiento de la velocidad relativa de meteorización de peces y mamíferos. Para ello se realizó una experimentación que consistió en la exposición de percas y oveja (Ovis aries) a condiciones de meteorización durante un período prolongado. Los resultados obtenidos fueron aplicados al conjunto ictioarqueológico del sitio Delta del Arroyo Vulcana I (DVI), en el cual el perfil anatómico demuestra la representación integral del esqueleto, pero la supervivencia diferencial de elementos óseos (Moreno y Svoboda 2013).

\section{MATERIALESY MÉTODOS}

El experimento se realizó en el predio del Centro Nacional Patagónico (Puerto Madryn, Chubut, Argentina). Se utilizó el esqueleto completo descarnado de cuatro percas de aproximadamente $43 \mathrm{~cm}$ de largo total y cuatro huesos largos de oveja. Se optó por colocar los ejemplares desarticulados y descarnados a fin de replicar condiciones de depositación cultural (procesamiento, consumo y descarte), lo cual imposibilitó establecer las etapas de desarticulación. Los restos se colocaron en una cuadrícula ( $\mathrm{m} \times \mathrm{I} \mathrm{m}$ ) dividida en cinco sectores: $\mathrm{A}$, B, C, D y Central. En los primeros cuatro sectores se colocó el cráneo descarnado de cada perca y en el Sector Central el resto del esqueleto (vértebras, costillas y espinas). Se colocó una jaula de igual dimensión que la cuadrícula para proteger los restos de la acción de animales carroñeros, lo que a su vez dificultó la pérdida de elementos por la acción del viento.

El contexto geomorfológico donde se realizó el experimento corresponde a una duna parcialmente vegetada cuya matriz sedimentaria está compuesta por granos de tamaño arena y algunas gravas. Este contexto es propicio para que los restos sean rápidamente enterrados. Dado que el objetivo de la experimentación fue explorar la supervivencia de elementos por procesos de meteorización subaérea, el tiempo de enterramiento no fue totalmente controlado. En este sentido, no se dispuso de un dispositivo para evitar la sedimentación y en algunos casos se registró este proceso mediante fotografías (ver más adelante).

Químicamente el sustrato donde están incluidos los huesos presenta un $\mathrm{pH}$ alcalino, lo cual favorece su preservación. La parcela donde se realizó el experimento no tiene pendiente, por lo que la dispersión horizontal por efecto gravitatorio es prácticamente nula. También se registraron los 
valores medios anuales de variables atmosféricas (temperatura, precipitación acumulada, radiación solar y magnitud del viento) obtenidas por la estación meteorológica del CENPAT.

Cada cinco meses se efectuó un monitoreo que consistió en fotografías tomadas desde un mismo punto. A los 36 meses se extrajo una muestra de especímenes parcialmente enterrados correspondientes a vértebras $(n=29)$, espinas de la aleta dorsal y anal $(n=17)$ y costillas $(n=30)$, con el fin de realizar una primera comparación con los restos ictioarqueológicos (Moreno y Pérez Ruiz 2010). El criterio de selección de estos elementos se basó en su alta abundancia en ambos conjuntos (experimental y arqueológico). A los 67 meses finalizó el experimento y se recuperaron todos los especímenes a partir de una excavación tradicional con el empleo de zaranda fina ( $1 \mathrm{~mm}$ de apertura de malla). Los restos ícticos recuperados fueron analizados en el laboratorio donde se observó la superficie ósea de forma macroscópica y con aumentos de 10 a 50x (lupa binocular ZEIZZ STEMI 2000 C).

El análisis de los resultados de la
experimentación consistió en dos pasos
metodológicos: por un lado, la reconstrucción de la secuencia de meteorización de algunos restos a partir de observaciones fotográficas; por el otro, el análisis de la totalidad de los especímenes recuperados en la excavación. El primer paso consistió en la observación de fotografías de los sectores A y Central para estimar la secuencia de meteorización a partir de la caracterización de atributos como la exfoliación, agrietamientos y fragmentación (pérdida de masa ósea) (cf. Zangrando 2009). Dado que los restos fueron paulatinamente incorporados al médano (ver más adelante) deteniéndose así el avance de la destrucción ósea (Behrensmeyer 1978), no se pudieron realizar observaciones de la secuencia total de destrucción de los huesos, por lo cual no se definieron estadios de meteorización. Además, la observación de fotografías permitió registrar la secuencia de sedimentación de algunos elementos. Para ello se consideró que un hueso está parcialmente enterrado cuando el $30 \%$ a $90 \%$ de su superficie se encuentra cubierto por sedimento; $y$ totalmente enterrado cuando el sedimento cubre más del $90 \%$ del hueso. Es importante mencionar que el registro fotográfico presentó una serie de limitaciones. En primer lugar no permitió visualizar con claridad la secuencia de varios elementos como vómer, postemporal, premaxilar, angular, cuadrado, epihial, basioccipital y otolito. Por otra parte, sólo posibilitó fotografiar la cara expuesta de cada elemento.

El segundo paso metodológico implicó el análisis del total de restos recuperados en la excavación. Para estimar la pérdida de masa ósea se calculó la completitud del elemento, definido como el porcentaje representado del elemento completo; y luego, se obtuvo el valor medio de completitud (cf. Nicholson 1992b). Se calculó el índice de supervivencia dividiendo el MNE observado sobre el esperado para cuatro percas. Para la cuantificación se empleó el MNE y MAU\% siguiendo la propuesta de Zangrando (2009). De forma exploratoria se compararon los perfiles anatómicos con los valores de densidad mineral ósea de perciformes y scorpaeniformes, extraídos de Falabella y coautores (1994), cuya estructura ósea es similar a la perca (Gosztonyi com. pers. 20I I).

La muestra ictioarqueológica proviene del sitio DVI (ca. I400 AP) ubicado geográficamente en la margen oeste del lago Musters, en el sector CentroSur de la Provincia de Chubut (Moreno y Pérez Ruiz 2010). Del total del conjunto arqueofaunístico los especímenes de peces representan un $26 \%$, entre los cuales el $11 \%$ corresponde a perca. En lo que respecta a las alteraciones antrópicas, se registraron evidencias de combustión en un $0,4 \%$ aunque no se observaron huellas de corte.Tampoco se observaron daños por corrosión gástrica o por masticado de carnívoros. Por estas evidencias y la información contextual se concluyó que el conjunto íctico fue generado por acción antrópica (Moreno y Svoboda 2013). 


\section{RESULTADOSY DISCUSIÓN}

Resultados de la experimentación

Como se observa en la Figura I los huesos observados en fotografías estuvieron expuestos durante 32 meses. Durante este período el cleitro, opercular, preopercular y dentario presentan signos de exfoliación mientras que en las espinas dorsales $y$ anales $y$ en el parasfenoides se observan agrietamientos longitudinales. La mayoría de los elementos fueron parcialmente enterrados entre los 32 meses y los 45 meses; a excepción de varias vértebras que continuaron expuestas hasta los 67 meses (final de la experimentación) (Figura I). Al comparar la secuencia de destrucción ósea entre la perca y la oveja se observó que los elementos de esta última se encuentran en el estadio I de meteorización (sensu Behrensmeyer 1978), en tanto que la superficie ósea de gran parte de los restos de peces se encuentra intensamente afectada.

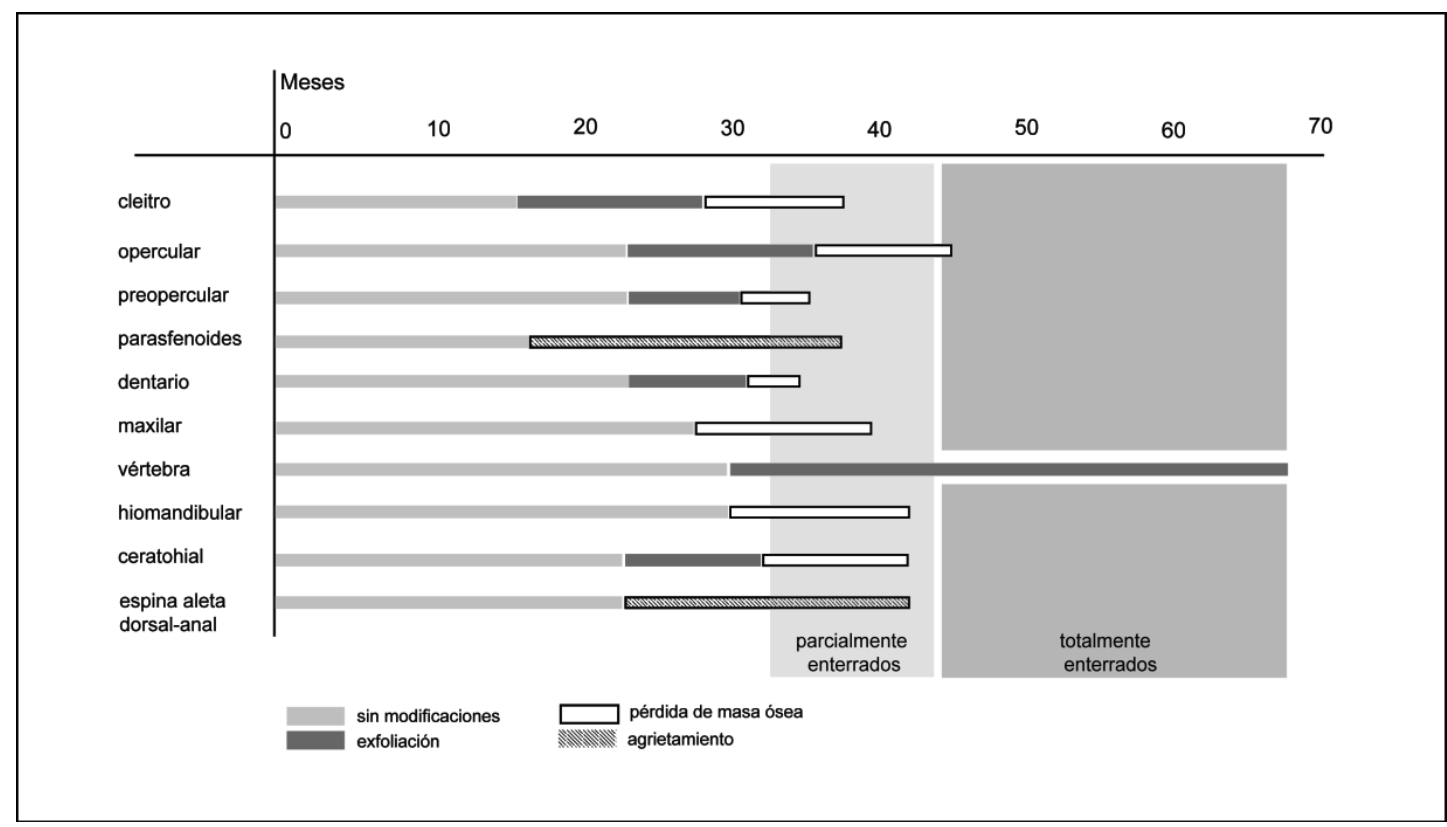

Figura 1: Secuencia preliminar de meteorización y sedimentación de algunos elementos óseos de P. trucha del conjunto experimental.

Figure 1: Preliminary weathering and sedimentation sequence of some P. trucha skeletal elements of the experimental set.

Con respecto al índice de supervivencia y la completitud se observan diferencias entre elementos (Tabla I). Los elementos otolito, basioccipital, vértebras, espina dorsal-anal, epihial y ceratohial muestran una alto índice de supervivencia y alta completitud. En el caso del otolito, esta resistencia a la meteorización podría deberse a la "dureza" de este elemento, el cual se caracteriza por una concreción formada por carbonato cálcico cristalizado en aragonito. En el basioccipital y las vértebras la morfología es un factor que podría influir en su alta supervivencia (Binford y Bertram 1977). Si bien estos últimos elementos presentan baja densidad mineral ósea (Falabella et al. 1994: Figura 5), su forma redondeada implica menor superficie de contacto con los agentes químicos y físicos de la meteorización. En cuanto al ceratohial y epihial, que presentan formas planas y por lo tanto mayor superficie de contacto, la forma no parece ser una explicación válida para su alta supervivencia. 


\begin{tabular}{|c|c|c|c|c|c|c|}
\hline Elemento & $\begin{array}{c}\text { MNE } \\
\text { observado }\end{array}$ & $\begin{array}{c}\text { MNE } \\
\text { esperado }\end{array}$ & $\begin{array}{c}\text { Índice de } \\
\text { supervivencia }\end{array}$ & Ranqueo & $\begin{array}{c}\text { Valor de } \\
\text { completitud }\end{array}$ & Ranqueo \\
\hline Otolito & 7 & $7 *$ & । & । & 100 & 1,25 \\
\hline Ceratohial & 7 & 8 & 0,87 & 2 & 100 & 1,25 \\
\hline Basioccipital & 3 & 4 & 0,75 & 3 & 91,6 & 2 \\
\hline Vértebra caudal y precaudal & 100 & 134 & 0,74 & 4 & 80 & 7 \\
\hline Espina de aleta dorsal y anal & 28 & 38 & 0,73 & 5 & 91 & 3 \\
\hline Epihial & 5 & $7 *$ & 0,71 & 6 & 90 & 4 \\
\hline Cuadrado & 3 & 8 & 0,37 & 7 & 100 & 1,25 \\
\hline Pterigióforos & 10 & 36 & 0,27 & 8 & 85 & 6 \\
\hline Parasfenoides & । & 4 & 0,25 & 9,2 & 100 & 1,25 \\
\hline Hiomandibular & 2 & 8 & 0,25 & 9,2 & 62,5 & 9,5 \\
\hline Angular & 2 & 8 & 0,25 & 9,2 & 62,5 & 9,5 \\
\hline Dentario & 2 & 8 & 0,25 & 9,2 & 55 & 10 \\
\hline Premaxilar & 2 & 8 & 0,25 & 9,2 & 87,5 & 5 \\
\hline Opercular & I & 8 & 0,12 & 10,25 & 25 & 12,5 \\
\hline Maxilar & I & 8 & 0,12 & 10,25 & 75 & 8 \\
\hline Cleitro & I & 8 & 0,12 & 10,25 & 37,5 & 11 \\
\hline Preopercular & I & 8 & 0,12 & 10,25 & 25 & 12,5 \\
\hline Vómer & 0 & $2 *$ & 0 & 11,5 & 0 & 13,5 \\
\hline Postempotral & 0 & 6* & 0 & 11,5 & 0 & 13,5 \\
\hline
\end{tabular}

Tabla 1: Índice de supervivencia y de completitud de los elementos óseos de P. trucha luego de estar expuestos a condiciones de meteorización. ${ }^{*}$ : Debido a que algunos elementos no se incorporaron al experimento, el MNE esperado es menor que el correspondiente a cuatro percas.

Table 1: Survival index and completeness of P. trucha bones elements after exposure to weathering conditions. *: Since some items were not incorporated to the experiment, the expected MNE is less than four perches.

El resto de los elementos craneales (cuadrado, parasfenoides, hiomandibular, angular, dentario, premaxilar, opercular, maxilar, cleitro y preopercular) y apendiculares (pterigióforos) tuvieron una supervivencia baja (Tabla I). Particularmente el vómer y el postemporal desaparecieron completamente. La mayoría de estos elementos presentan formas planas y/o ramificaciones por lo cual la superficie de contacto con agentes químicos y físicos es mayor, haciéndolos susceptibles a la destrucción (Falabella et al. 1994). En concordancia con los valores del índice de supervivencia, la completitud de cada uno de estos elementos es moderada a baja (Tabla I). Se presentan algunas excepciones como el cuadrado, parasfenoides, maxilar y premaxilar que si bien su expectativa de supervivencia es baja, los pocos elementos que sobrevivieron no sufrieron pérdida de masa ósea.

En cuanto a la densidad mineral ósea, no hay relación entre este factor y la abundancia relativa de los elementos del conjunto experimental (Tabla 2). Si bien no disponemos de los valores densitométricos de perca, en principio la densidad ósea no sería un factor determinante para la supervivencia diferencial de elementos óseos bajo condiciones de meteorización subaérea. Esto es acorde a las conclusiones alcanzadas por Nicholson en los experimentos sobre los efectos de la meteorización en peces (Nicholson 1992b:Tabla 3). 


\begin{tabular}{|c|c|c|c|c|c|c|}
\hline \multirow[b]{2}{*}{ Elemento anatómico } & \multicolumn{2}{|l|}{ MAU\% } & \multicolumn{4}{|c|}{ g/cm3 (Falabella et al. 1994) } \\
\hline & Conjunto experimental & $\begin{array}{c}\text { Delta del Arroyo } \\
\text { Vulcana I }\end{array}$ & $\begin{array}{c}\text { Sebastes } \\
\text { capensis } \\
\text { (a) }\end{array}$ & $\begin{array}{c}\text { Pinguipes chilensis } \\
\text { (b) }\end{array}$ & $\begin{array}{c}\text { Cheilodactylus } \\
\text { variegatus } \\
\text { (c) }\end{array}$ & $\begin{array}{c}\text { Trachurus } \\
\text { symmetricus (d) }\end{array}$ \\
\hline Angular & 28,5 & 30 & 1,24 & 1,29 & 1,45 & 1,15 \\
\hline Cuadrado & 42,8 & 90 & 1,44 & ।,35 & 1,39 & 1,26 \\
\hline Dentario & 28,5 & 20 & 1,42 & 1,28 & 1,36 & 1,04 \\
\hline Hiomandibular & 28,5 & 10 & 1,43 & 1,09 & 1,38 & 1,1 \\
\hline Maxilar & 14,2 & 40 & 1,58 & 1,55 & $|, 5|$ & ।,56 \\
\hline Opercular & 14,2 & 30 & 1,53 & 1,4 & 1,61 & 1,15 \\
\hline Premaxilar & 28,5 & 30 & 1,38 & 1,38 & 1,53 & 1,22 \\
\hline Preopercular & 14,2 & 30 & $1,4 \mid$ & 1,29 & 1,61 & 1,06 \\
\hline Vértebra & 82,8 & 65 & 1,3 & 1,36 & 1,37 & 0,98 \\
\hline Otolito & 100 & 90 & 2,77 & 2.58 & 2,78 & 2,55 \\
\hline Ceratohial & 100 & 90 & - & - & - & - \\
\hline Basioccipital & 85 & 100 & - & - & - & - \\
\hline $\begin{array}{l}\text { Espina aleta dorsal y } \\
\text { anal }\end{array}$ & 68,5 & 100 & - & - & - & - \\
\hline Epihial & 71,4 & 60 & - & - & - & - \\
\hline Pterigióforos & 4,2 & 37 & - & - & - & - \\
\hline Parasfenoides & 28,5 & 20 & - & - & - & - \\
\hline Cleitro & 14,2 & 30 & - & - & - & - \\
\hline Preopercular & 14,2 & 30 & - & - & - & - \\
\hline Vómer & 0 & 40 & - & - & - & - \\
\hline \multirow[t]{3}{*}{ Postempotral } & 0 & 20 & - & - & - & - \\
\hline & \multicolumn{2}{|c|}{ MAU\% conjunto experimental contra: } & $\begin{array}{l}\mathrm{g} / \mathrm{cm} 3(\mathrm{a}), \\
\mathrm{rs}=-0,05 ; \mathrm{ns}\end{array}$ & $\begin{array}{l}\mathrm{g} / \mathrm{cm} 3(b) \\
\mathrm{rs}=0,08 ; \mathrm{ns}\end{array}$ & $\begin{array}{l}\mathrm{g} / \mathrm{cm} 3(\mathrm{c}) \\
\mathrm{rs}=-0,23 ; \mathrm{ns}\end{array}$ & $\begin{array}{l}\mathrm{g} / \mathrm{cm} 3(\mathrm{~d}) \\
\mathrm{rs}=0,09 ; \mathrm{ns}\end{array}$ \\
\hline & \multicolumn{2}{|c|}{ MAU\% Delta del Arroyo Vulcana I contra: } & $\begin{array}{l}\mathrm{g} / \mathrm{cm} 3(\mathrm{a}), \\
\mathrm{rs}=-0,32 ; \mathrm{ns}\end{array}$ & $\begin{array}{l}\mathrm{g} / \mathrm{cm} 3(b) \\
\mathrm{rs}=0,69 ; \mathrm{ns}\end{array}$ & $\begin{array}{l}\mathrm{g} / \mathrm{cm} 3(\mathrm{c}), \\
\mathrm{rs}=-0,30 ; \mathrm{ns}\end{array}$ & $\begin{array}{l}\mathrm{g} / \mathrm{cm} 3(\mathrm{~d}), \\
\mathrm{rs}=-0,50 ; \mathrm{ns}\end{array}$ \\
\hline
\end{tabular}

Tabla 2: MAU\% de P. trucha del conjunto experimental y DV1 contra los valores densitométricos de perciformes y scorpaeniformes. Nota: rs: coeficiente de correlación Spearman; ns: no significativo.

Table 2: MAU\% frequencies of P. trucha in the experimental and DV1 assemblages against perciform and scorpaeniform densitometric values. Note: rs: Spearman's correlation; ns: not significant. 
Aplicación de los resultados de la experimentación al conjunto ictioarqueológico DVI

No hay relación entre la densidad mineral ósea y la abundancia relativa de los elementos del conjunto DVI (Tabla 2). En cambio se observa una correlación significativa (aunque de intensidad media), entre el perfil anatómico de DVI y el índice de supervivencia (coeficiente de correlación Spearman= 0,65; $p<0,05$ ) (Figura 2). En principio, esto sugiere la incidencia de la meteorización en la supervivencia diferencial en DVI; no obstante, otras consideraciones desestiman la importancia de este proceso. Por un lado, la presencia de elementos de baja expectativa de supervivencia (e.g. vómer, cleitro, maxilar, opercular) señalan un proceso de enterramiento rápido de los huesos; por el otro, los especímenes presentan un buen estado de conservación: solo I8 (I,9\%) especímenes presentaron exfoliaciones y agrietamientos en las superficies óseas. Estos atributos se presentaron en las carillas de vértebras, espinas dorsal-anal y en una cara del ceratohial y del epihial (Moreno y Svoboda 20I3). En este sentido, los restos se habrían enterrado relativamente rápido en un período aproximado de I a 20 meses. En suma, la correlación de intensidad media- entre el perfil anatómico de DVI y el índice de supervivencia se trataría de un problema de equifinalidad.

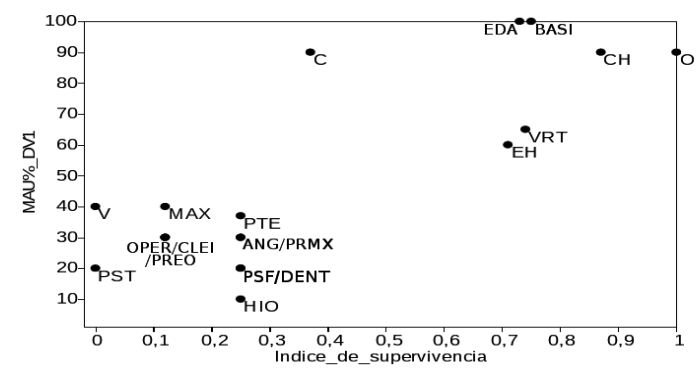

Figura 2: Relación entre el índice de supervivencia y MAU\% de DV1 (Spearman rs: 0,65; p < 0,05). O: otolito; $\mathrm{CH}$ : ceratohial; BASI: basioccipital; VRT: vértebras caudales y precaudales; EDA: espina de aleta dorsal y anal; EH: epihial; C: cuadrado; PSF: parasfenoides HIO: hiomandibular; ANG: angular; DENT: dentario; PRMX: premaxilar; OPER: opercular; MAX: maxilar; CLEI: cleitro; PREO: preopercular; V:vómer; PST: postemporal; PTE: pterigióforo.

Figure 2: Relationship between survival index and MAU\% frequencies of DV1 (Spearman rs: 0.65, $p$ <0.05). O: otolith; $C H$ : ceratohyal; BASI: basioccipital; VRT: caudal and precaudal vertebrae; EDA: dorsal and anal fin spine; EH: epihial; C: quadrate; PSF: parasphenoid;

HIO: hyomandibula; ANG: angular; DENT: dentary; PRMX: premaxilla; OPER: opercular; MAX: maxilla; CLEI: cleithrum; PREO: preopercular; V: vomer; PST: postemporal; PTE: pterygiophore.

\section{CONSIDERACIONES FINALES}

La meteorización afectó de manera diferencial la supervivencia de elementos óseos de las percas experimentales. Las unidades anatómicas que presentan mayor potencialidad de supervivencia corresponden tanto al cráneo (otolito, basioccipital, epihial y ceratohial) como al poscráneo (vértebras y espina dorsal y anal). En los contextos arqueológicos, su presencia y la ausencia de los demás huesos craneales y poscraneales indicarían que los peces entraron completos y que, posteriormente, la meteorización tuvo una alta incidencia sobre los restos óseos. En principio, la densidad mineral ósea no es un factor que media en la supervivencia de partes esqueletales en las muestras experimental y arqueológica, aunque en un futuro deberán establecerse los valores de la densidad mineral ósea de perca para una mejor aproximación.

En otro orden, los resultados de la experimentación demostraron que la velocidad de meteorización en peces es más rápida que la registrada en oveja, lo cual advierte sobre posibles sesgos tafonómicos para determinar la presencia íctica en los conjuntos arqueofaunísticos.

En cuanto a la aplicación de los resultados obtenidos de la experimentación al conjunto ictioarqueológico de DVI, se constató que la meteorización no afectó significativamente la supervivencia de los elementos óseos; en este sentido, es probable que la rápida sedimentación de los restos haya detenido el avance de este proceso. Es interesante destacar que las condiciones ambientales del lago Musters son más extremas que en la costa marina, que es donde se realizó el experimento. En suma, la representación diferencial de elementos anatómicos observada en DVI debería vincularse con otros factores aún no estudiados (e.g. procesos diagenéticos, consumo, descarte).

Finalmente, cabe destacar que el experimento no tuvo como objetivo establecer una escala de meteorización similar a la establecida para mamíferos (cf. Behrensmeyer 1978), de este modo se permitió el enterramiento de los restos. Es por ello, que los resultados aquí obtenidos son aplicables a un intervalo de tiempo (i.e. duración de la meteorización) acotado a los 42 meses, lapso en que los restos estuvieron expuestos y parcialmente enterrados. Como agenda futura nos 
proponemos realizar experimentos que contemplen la desarticulación de las carcasas y el establecimiento de estadios de meteorización para restos óseos de peces, especialmente de perca.

\section{AGRADECIMIENTOS:}

Este trabajo fue posible a los subsidios PIPCONICET 072I y PI UNSPSJ 0II/09, y a una beca doctoral Tipo I CONICET. A Gonzalo Lana, Atila Gosztonyi, Daniela Robledo y nuestros compañeros de equipo. A los evaluadores anónimos, a Francisco Zangrando y Julieta Gómez Otero cuyos comentarios y sugerencias ayudaron a mejorar el trabajo.

\section{BIBLIOGRAFÍA}

Acosta, A, D. Loponte y J. Musali. 2007. "A Taphonomic Approach to the Icthyoarchaeological assemblage of La Bellaca site 2, Wetland of the Lower Paraná River, Pampean Region (Argentina)". En Taphonomy and Zooarchaeology in Argentina, editado por M. Guitiérrez, G. Barrientos, M. Salemme, L Miotti y G Mengoni Goñalons, pp: 7I-88. British Archaeological Reports, International Series, Oxford.

Binford, L. y J. Bertram. 1977. "Bone frequencies and attritional processes”. En For Theory Building in Archaeology, editado por L.R. Binford, pp:77-I53.Academic Press, New York.

Behrensmeyer, A.K. 1978. "Taphonomic and ecological information from bone weathering”. Paleobiology 4:150-162.

Borrero, L. A. 2007. "Longitudinal taphonomic studies in Tierra del Fuego, Argentina”. En Taphonomy and Archaeozoology in Argentina, editado por M. Gutierrez, G. Barrientos., M. Salemme, L Miotti y G Mengoni Goñalons, pp: 219-233. British Archaeological Reports, International Series, Oxford.

Butler, V.L. 1993. "Natural Versus Cultural Salmonid Remains: Origin of The Dalles Roadcut Bones, Columbia River, Oregon, U.S.A." Journal of Archaeological Science 20: I-24.
Cruz, I. 2008. "Avian and mammalian bone taphonomy in southern continental Patagonia: a comparative approach". Quaternary International 180:30-37.

Falabella, F., M. L. Vargas y R. Meléndez. 1994. "Differential preservation and recovery of fish remains in Central Chile. Fish Exploitation in the Past". En Proceeding of the $7^{\text {th }}$ meeting of the ICAZ. Fish Remains Working Group, editado por Van Neer, pp: 25-35. Annales du Musée Royal de l'Afrique Centrale, Sciences Zoologiques, Tervuren.

Gifford-Gonzalez, D., K. M. Stewart y N. Rybczynski. 1999. "Human Activities and Site Formation at Modern Lake Margin Foraging Camps in Kenya". Journal of Anthropological Archaeology 18: 397-440.

Moreno, J.E. y H. Pérez Ruiz. 2010. “Evidencias de utilización prehispánicas de recursos fluviales en la cuenca del lago Musters (Chubut, Argentina)". Actas del XVII Congreso Nacional de Arqueología Argentina, Tomo I, pp: 345-350. Mendoza, Argentina.

Moreno, J.E. y A. Svoboda. 20I3. Explotación de peces y guanacos en el interior de Patagonia central: aportes del sitio Delta del Arroyo Vulcana I (lago Musters, Chubut). Manuscrito en posesión de los autores.

Nicholson, A. R. 1992a. "Bone Survival: the Effects of Sedimentary Abrasion and Trampling on Fresh and Cooked Bone". International Journal of Osteoarchaeology 2: 79-90.

---- 1992b. "An Assessment of the Value of Bone Density Measurements to Archaeoichthyological Studies". International Journal of Osteoarchaeology 2:139-154.

---- 1992-1993. “An Investigation into the effects on fish bone of passage trough the human gut: some experiments and comparisons with archaeological material”. Circaea I0: 38-5I.

Richter, J. 1986. "Experimental study of heat induced morphological change in fish bone collagen". Journal of Archaeological Science I3:477-48I.

Stewart, M. K y D. Gifford-Gonzalez. 1994. “An Ethnoarchaeological Contribution to Identifying Hominid Fish Processing Sites". Journal of Archaeological Science 21:237-248.

Zangrando, A.F. 2009. Historia evolutiva, tiempos y subsistencia humana en la región del Canal Beagle. Una aproximación zooarqueológica. Sociedad Argentina de Antropología.

Zohar, I., T. Dayan, E. Galili y E. Spanier.200I.“Fish Processing During the Early Holocene: a Taphonomic Case Study from Coastal Israel". Journal of Archaeological Science 28: I04 I- 1053. 